\title{
Neuralgia-inducing cavitational osteonecrosis (NICO)
}

Bouquot James E, Roberts AM, Person P, Christian J

\section{Source}

Bouquot JE, Roberts AM, Person P, Christian J. (1992). Neuralgia-inducing cavitational osteonecrosis (NICO). Oral Sugery Oral Medicine Oral Pathology, vol. 73:307-19

Chronic facial neuralgia induced by intraosseous cavity formation and long-standing bone necrosis with minimal healing 